\title{
CHARACTERIZATION OF SURFACE MORPHOLOGY AND ITS CORRELATION WITH FRICTION PERFORMANCE OF BRAKE PADS
}

\author{
P. D. Neis ${ }^{1}$, N.F. Ferreira ${ }^{1}$, J. Sukuraman ${ }^{2}$,P.D. Baets ${ }^{2}$, M. Andó ${ }^{3}$, L. T. Matozo ${ }^{4}$, D. Masotti ${ }^{4}$ \\ ${ }^{1}$ Universidade Federal do Rio Grande do Sul, Laboratory of Tribology, Brazil \\ ${ }^{2}$ Ghent University, Laboratory Soete, Belgium \\ ${ }^{3}$ Savaria Institute of Technology, Department of Mechanical Engineering, Hungary \\ ${ }^{4}$ Fras-le S.A., Rod RS-122, 10945 - km 66, Caxias do Sul, Brazil.
}

\begin{abstract}
The present work presents the morphology evolution of a brake material surface submitted to braking tests through a laboratory-scale tribometer. Optical microscope images of the material's surface were obtained for every 10 braking operations. These images were post-processed in appropriate computational software. By means of the image segmentation technique, morphological parameters related to the brake material surface were estimated. The wear rate and also the coefficient of friction resulting from the tests were measured. For the NAO material used in this study, the friction behaviour revealed to be strongly associated with the amount of contact plateaus. Besides, the mean area of the contact plateaus was the main factor responsible for increasing the real contact area of the friction material. The higher wear rate observed in the first braking operations can be mainly attributed to the higher surface roughness measured in this condition. As the braking operations progress, the plateaus becomes flatter and wear rate is reduced. Finally, the image segmentation technique proved adequate for investigating morphological aspects in friction material surface.
\end{abstract}

Keywords: brake pad; image segmentation; real contact area

\section{INTRODUCTION}

Nowadays there is an increasing number of researches devoted to study aspects related to the surface morphology of brake materials (brake pads and linings). For instance, some publications describe the mechanism of formation and destruction of the contact plateaus, which are the elements that effectively make contact between a friction pair (brake pad and disc). Yet, according to these studies, under certain circumstances the existence of large amount of small loose wear particles (also known as wear debris or third body) passing through the contact entrapped and embedded into pad interface due to the applied pressure, shear force and friction heat. Unevenly distributed wear and compaction of wear debris results in a surface characterised by flat plateaus, rising above the rest of the surface. As explained by Eriksson [1], the area of real contact is confined within the contact plateaus.

On the other hand, Ostermeyer and Wilkening [4] describe the mechanism of destruction of the contact plateaus which are formed on the surface of a brake composite material. According to this study, a contact plateau might be destroyed when its size becomes too large. Third body abrasion and erosion by wear debris transported through the contact are the processes which lead to the contact plateaus destruction.

Although many authors have focused on explaining the mechanism of formation and destruction of the contact plateaus, relatively few researchers have addressed the relationship between the morphology of the plateaus and performance (friction and wear) of brake materials.

Thus, this work aims to correlate morphological aspects related to the brake material's surface (number of plateaus - N, mean area of plateaus - Ap, as well as the real contact area of the brake pad - Ar) with the coefficient of friction $(\mu)$ and wear resulting from braking tests. The experiments consisted of subjecting a non asbestos organic (NAO) brake material to a total of 50 braking operations, which were performed on a laboratory-scale tribometer. Optical microscope images of the material's surface were obtained at every 10 braking operations, a condition described as "quasi in situ situation". These images were post-processed in appropriate computational software. By means of the segmentation technique, Ar was estimated, as well as the $\mathrm{N}$ and Ap. Finally, correlations between coefficient of friction and morphological aspects of the brake pad were established and discussed throughout this paper. Results of wear rate and roughness complement the analysis. 


\subsection{The laboratory-scale tribometer}

Figure 1 shows a scheme of the laboratory-scale tribometer used for conducting the braking tests performed in the current study. This test rig has been especially developed in order to characterize friction materials used as brake pads. Precision of the friction measurements performed by the testing machine is $\pm 0,013$, as previously shown in Neis [5].

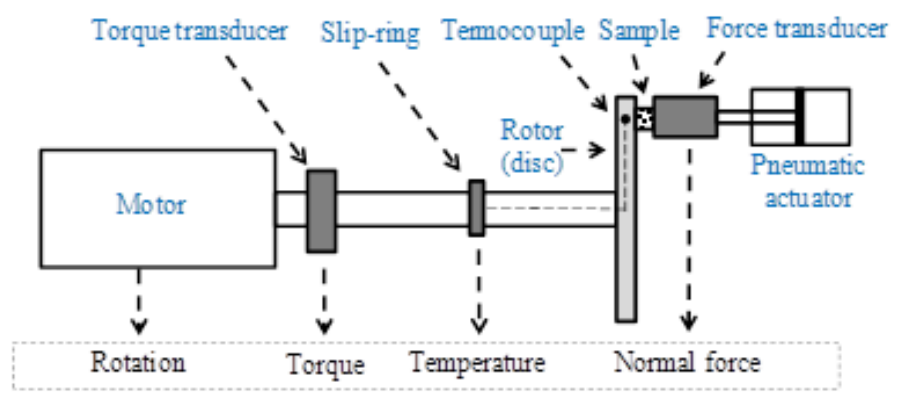

Figure 1. Scheme of the laboratory-scale tribometer.

\subsection{Samples preparation}

A friction material classified as "Non Asbestos Organic - NAO" was used in the braking tests. This material belongs to a brake pad which is employed in a passenger car in Brazil. In order to be used as a sample in the laboratory scale tribometer, this brake pad was machined into a cylindrical shape with diameter of 18 $\mathrm{mm}$ (nominal contact area of $254 \mathrm{~mm}^{2}$ ).

The disc used in the braking tests is made of grey cast iron, which is composed of $3-4 \% \mathrm{C}, 1-2 \% \mathrm{Si}$ and $\mathrm{Mn}$ and also $1 \%$ of trace elements. It has a diameter and thickness of $159 \mathrm{~mm}$ and $12 \mathrm{~mm}$, respectively. Before to the tests, the surface of the disc was abraded with sandpaper and a roughness Ra lower than $0,20 \mu \mathrm{m}$ was obtained. A K-type thermocouple was embedded $6 \mathrm{~mm}$ below the disc surface, at a radius of $40 \mathrm{~mm}$, which corresponds to the center of the friction track. This instrument was employed to measure the disc temperature in the experiments. The disc and sample used in the tests is shown in Figure 2.

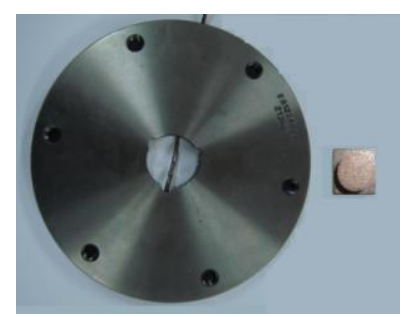

Figure 2. A brake disc (dia.=159 $\mathrm{mm}$ ) and a brake material sample used in the braking tests.

\subsection{Test procedure}

In this paper, a combination of friction measurements and optical examinations (microscopy) of the sample was performed. This allows to observe the evolution of the sample's surface morphology as the number of braking operations progresses. Table 2 summarizes the brake operating parameters used in the tests.

Table 2. Description of the test procedure used in this paper.

\begin{tabular}{|c|c|c|c|c|}
\hline $\begin{array}{c}\text { Contact } \\
\text { pressure }(\mathrm{Mpa})\end{array}$ & $\begin{array}{c}\text { Relative initial sliding } \\
\text { velocity }(\mathrm{m} / \mathrm{s})\end{array}$ & $\begin{array}{c}\text { Initial temperature } \\
\text { of the disc }\left({ }^{\circ} \mathrm{C}\right)\end{array}$ & $\begin{array}{c}\text { Microscopy performed at the } \\
\text { following braking operations }\end{array}$ & $\begin{array}{c}\text { Data acquisition } \\
\text { sampling rate }(\mathrm{Hz})\end{array}$ \\
\hline 4,7 & 7,7 & 100 & $10 / 20 / 30 / 40 / 50$ & 200 \\
\hline
\end{tabular}

\subsection{Microscopy}

The optical characterization of the sample's surface is performed with a stereo-microscope (CARL ZEISS ${ }^{\circ}$, model AxioLab A1, designed in Germany). It is equipped with a digital CCD camera with a resolution of 3.0 
Mega Pixel, which enables capturing images of the observed surface. Both top and side lights were used for capturing the microscope images. The analyzed area of each sample surface was focused on its center region and it has approximately $6,1 \mathrm{~mm} \times 4,4 \mathrm{~mm}\left(26,84 \mathrm{~mm}^{2}\right)$, as illustrated by Figure 3 . Moreover, a magnification of $50 \mathrm{X}$ was used for producing the micrographs.

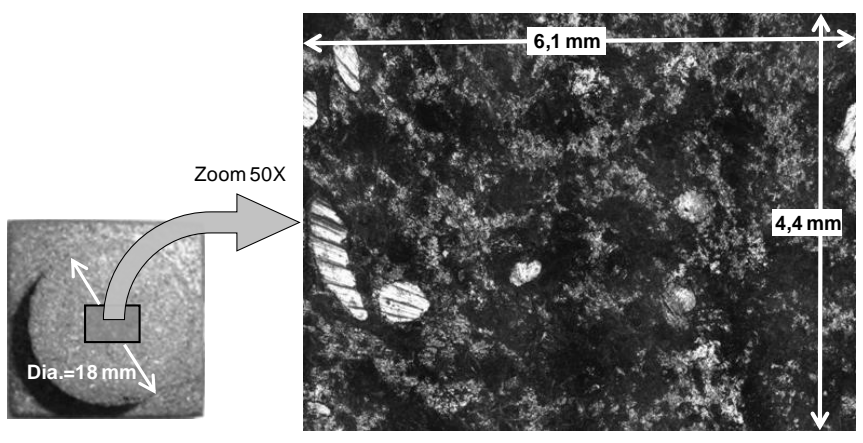

Figure 3. Illustration of a friction material sample and its corresponding area analyzed in the microscope.

The images obtained by microscope were processed by different softwares in two steps. Firstly, Photoshop CC software (Adobe Systems Software Ireland Ltd) was utilized to create the relatively large micrographs $(6,1 \mathrm{~mm} \times 4,4 \mathrm{~mm})$ showed in this study (e.g. in Fig. 3). It was made by assembling a set of 35 single micrographs, which were arranged in a grid of $7 \times 5$ images (wide $x$ height).

In a second step, segmentation technique based on Otsu's algorithm [6] was applied to the large micrographs, thereby allowing to study the morphology of the sample's surfaces. The software used in this case was the ImageJ (version 1.47v), which is an open source software. Figure 4 uses an exemplary micrograph to explain the segmentation technique. The picture in the left (Figure 4-a) corresponds to the micrograph before segmentation, while the picture in the right (Figure 4-b) represents the same micrograph subjected to the process of image segmentation. Micrograph corresponding to Figure 4-a reveals metal fibers on the material's surface, which correspond to the primary plateaus. It can be identified by their light and uniform coloration. The compacted debris appear in dark grey color, while the lowlands surrounding the plateaus appear as black. On the other hand, the processed micrograph (Figure 4-b) reveals just two colors, white and black. The first color (white) represents both the primary and secondary plateaus, while the last one (black) corresponds to the lowlands.

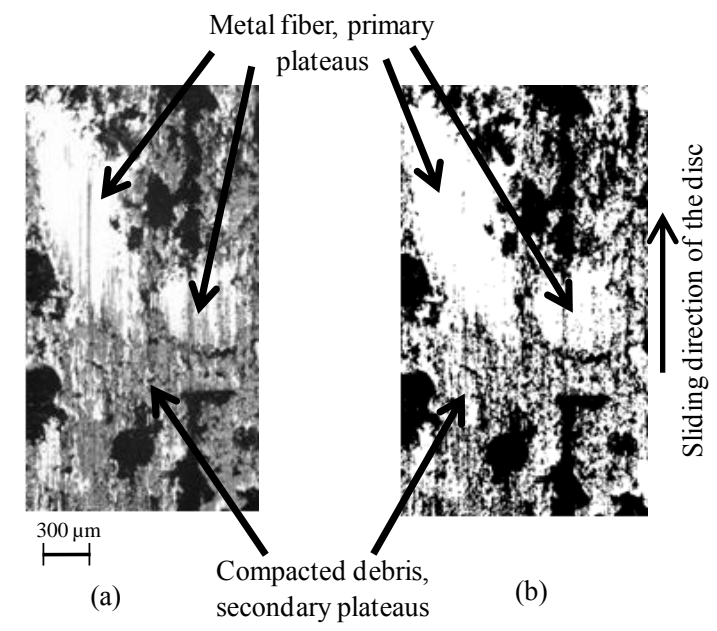

Figure 4. Micrograph of the NAO material: (a) before and (b) after segmentation technique..

\subsection{Methodology of the measurements}

The coefficient of friction $(\mu)$ plotted in all graphs represents the average of the last 3 applications corresponding to the analyzed braking number. For instance: the coefficient of friction of the 10th braking application is expressed as the average between braking 8,9 and 10. It is believed that the coefficient of friction determined in such manner is more reliable to the corresponding micrograph. As a result, a more accurate approximation between both the performance (coefficient of friction) and morphological data is achieved. 
An electronic scale with $\pm 0,2 \mathrm{mg}$ precision was used for weighing the brake sample. The weighing procedure was carried out for every 10 braking applications, allowing to observe the wear evolution.

The surface roughness of the friction material was evaluated with a portable surface roughness tester, also called rugosimeter (Mitutoyo, model SJ 201, Japan). The instrument was configurated to run in $\mathrm{Ra}$ parameter, with cutoff length of $0,8 \mathrm{~mm}$ and number of sampling lengths equal to 5 . The surface roughness of the friction material was determined for every 10 braking applications. Besides, this parameter was measured in both parallel and perpendicular directions with respect to the disc sliding. Three repetitions were carried out for each direction. So, data points plotted in the respective graph (see in section 'results and discussions') represent the average Ra roughness determined between the 3 measurements.

\subsection{The Person coefficient}

The Person's coefficient $(\rho)$ was used as an index in order to quantify the strength of association between the coefficient of friction and morphological characteristics of the sample's surface ( $A r, N$ and $A p)$. The intensity of $\rho$ was classified as: (i) $\rho \geq|0,70|$ for strong correlation; (ii) $|0,30|<\rho<|0,70|$ for mild correlation and (iii) $\rho \leq|0,30|$ for weak correlation. The numbers are expressed in absolute units because $\rho$ can assume either a positive or a negative value, depending on whether the correlation is positive or negative.

\section{$3 \quad$ RESULTS AND DISCUSSIONS}

Figure 5 presents the evolution of both $\mu$ and Ar versus the number of braking operations. Notice that $\mathrm{Ar}$ is expressed as a fraction (percentage) of the nominal contact area. Other authors - e.g. Eriksson [1], Yoon et al. [7] - also represented $\mathrm{Ar}$ in such a way.

The friction behavior, characterized as mean magnitude \pm standard deviation ( $\mu \mathrm{m} \pm$ std $\mu$ ), is $0,348 \pm 0,003$. Based on this result, the friction performance of the sample can be considered satisfactory, since relatively low variation in friction magnitude is observed. According to Blau [8], a good friction material to be used as brake pad must have a stable friction behavior. Yet, it is observed a stable behavior since the first braking operations (braking numbers 10 and 20). It suggests that the friction material selected in this study is not prone to show a strong running-in or bedding-in effect, which is characterized by a transitory increase in the friction magnitude when the sample wears out locally to conform to the disc surface.

On the other hand, a continuous growth in $\mathrm{Ar}$ is observed as the braking operations progress. The percentage of $\mathrm{Ar}$ changed from around $15 \%$ for the unused material (zero point in the graph) to approximately $45 \%$ after 50 braking operations. It represents an increase by a factor of 3 in Ar. Finally, such considerable variations in Ar suggest that the NAO material is sensitive to changes in its morphological characteristics.

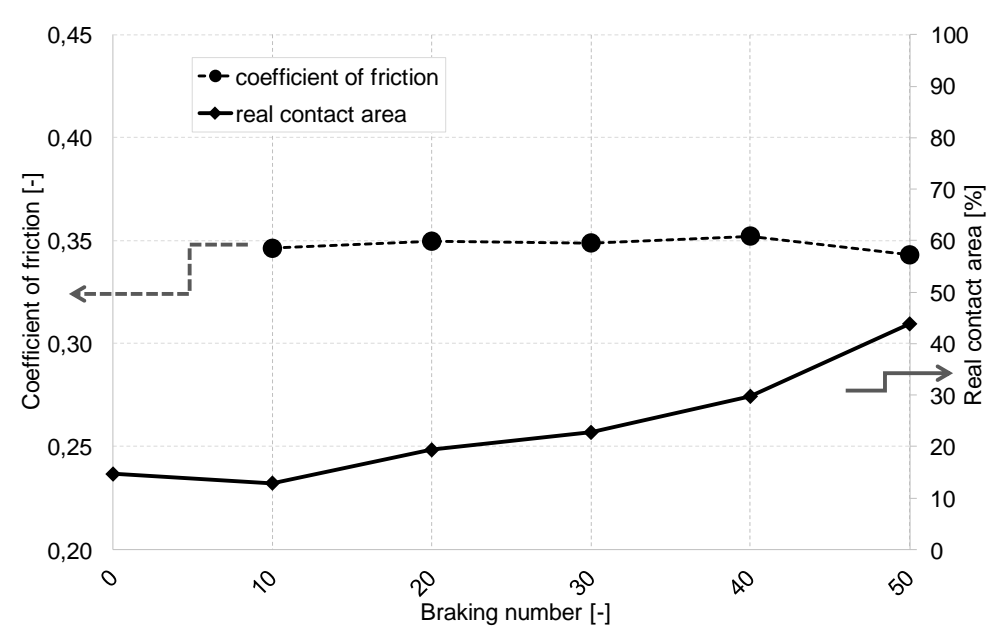

Figure 5. Evolution in friction and real contact area versus braking operation.

Figure 6 presents the evolution in the amount (number) and size (mean area) of the plateaus resulted from the tests. Regarding the number of plateaus, it is observed a relatively dispersive behavior. Results of Ap reveal a clear tendency of growing this parameter as the number of braking operations increase. The mean area of plateaus changed from around $100 \mu \mathrm{m}^{2}$ to $400 \mu \mathrm{m}^{2}$, considering the results from braking number 0 (unused material) and 50, respectively. Such differences represent an increase by a factor of 4 in Ap. 

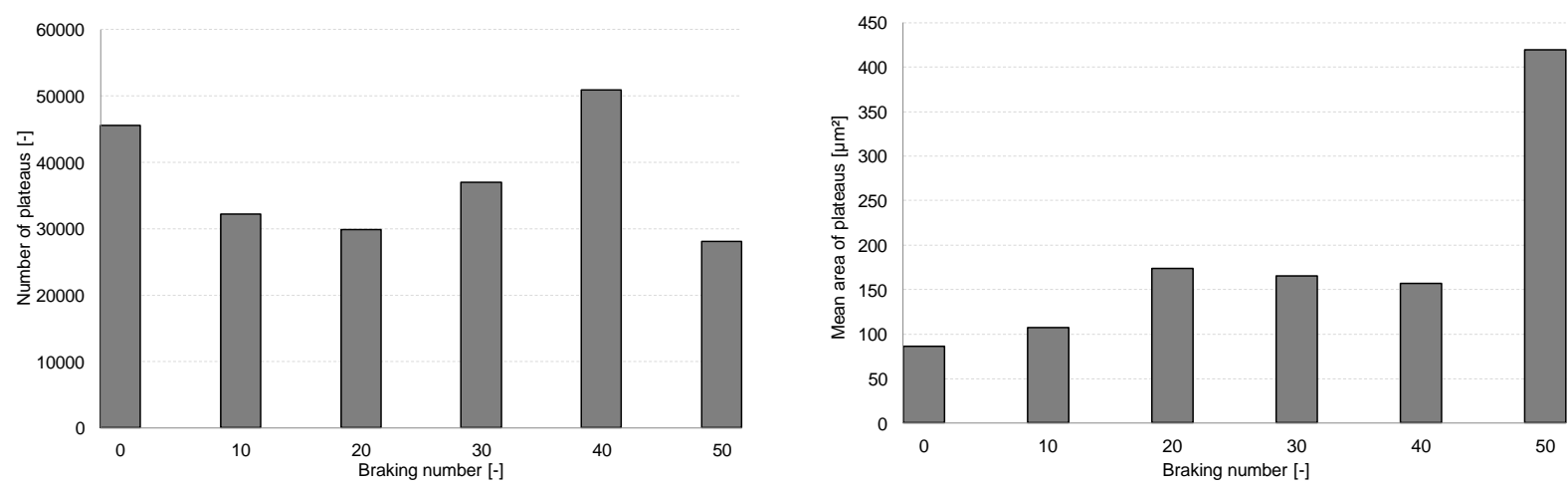

Figure 6. Evolution in number (left) and mean area of the contact plateaus (right).

The correlation results between morphological parameters and friction is shown in Table 3 . In order to make this table clearer, a gray color is used to fill the background of the corresponding cell with strong correlation. A positive and strong correlation was found between $\mathrm{N}$ and $\mu(+0,77)$. It suggests the friction behaviour is strongly associated with the amount of plateaus for the NAO material used in this study. Moreover, the strong and positive correlation between the $\operatorname{Ap}$ and the $\operatorname{Ar}(+0,92)$ indicates that the size of the plateaus is responsible for increasing $\mathrm{Ar}$ in the NAO material.

Table 3. Correlation results between friction and morphological parameters.

\begin{tabular}{|l|c|c|}
\hline & Coefficient of friction $-\mu$ & Real contact area - Ar \\
\hline Real contact area - Ar & $-0,38$ & - \\
\hline Total number of plateaus - N & 0,77 & $-0,17$ \\
\hline Mean area of plateaus - Ap & $-0,69$ & 0,92 \\
\hline
\end{tabular}

Figure 7 shows the micrographs taken for every 10 braking operations. The sequence in this figure starts by the zero point, corresponding to the unused friction material. It can be observed the increase of regions in white and light grey color. These regions correspond to the metallic fibers (primary plateaus) and compacted debris (secondary plateaus), which added make up the real contact area. Besides, it is possible to observe the growth of the contact plateaus as the braking operations progress.
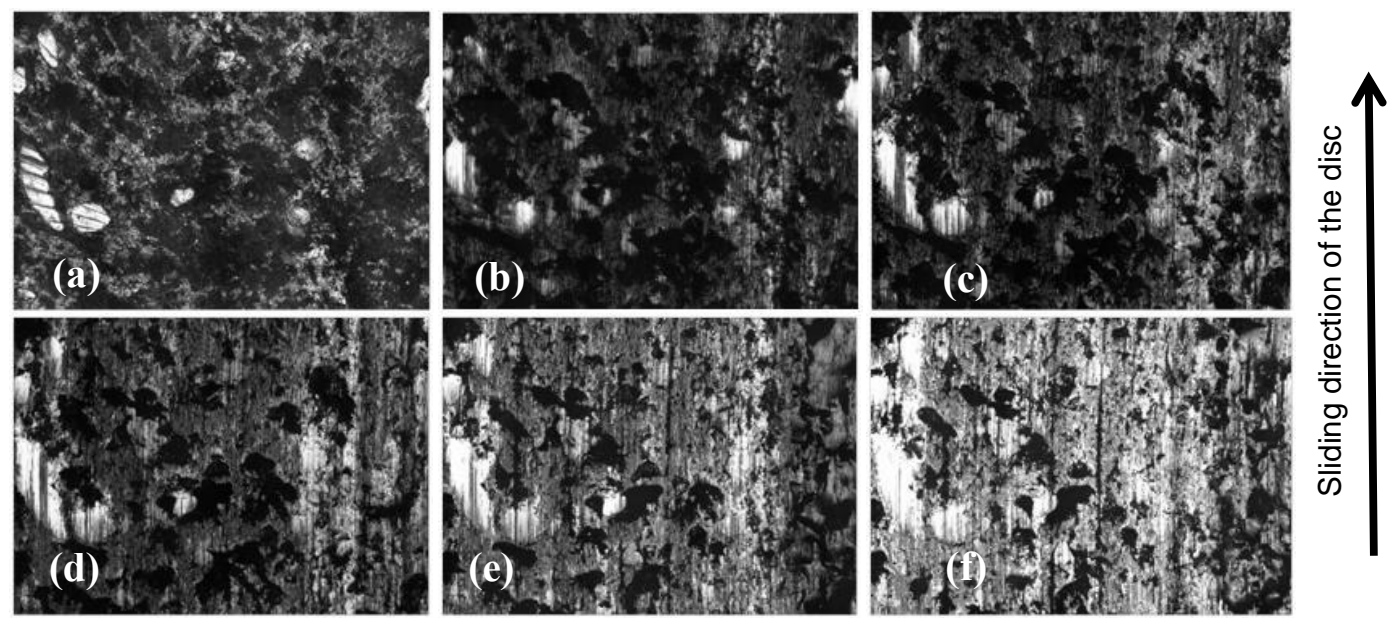

Figure 7. Micrographs of the (a) unused material and after braking number (b) 10, (c) 20, (d) 30, (e) 40, (f) 50 .

Figure 8 shows results of roughness (left) and wear (right) measured in the tests. Based on the roughness data, it is concluded that the surface of the friction material becomes smoother as the braking operations progress. No clear differences are observed between the roughness obtained with respect to the parallel and perpendicular directions to the disc sliding. Graph of the mass reveals that the wear rate is more 
pronounced in the first braking operations (braking numbers 10 and 20). It occurred because in the first braking operations the surface topography is characterized by higher peaks (higher Ra). These higher peaks are prone to be broken or degraded, resulting in higher wear rate. But, as the braking operation progress (braking number 30, 40 and 50), the peaks become smaller (Ra is lower) and flatter (higher Ap). In this moment, the wear rate is reduced.
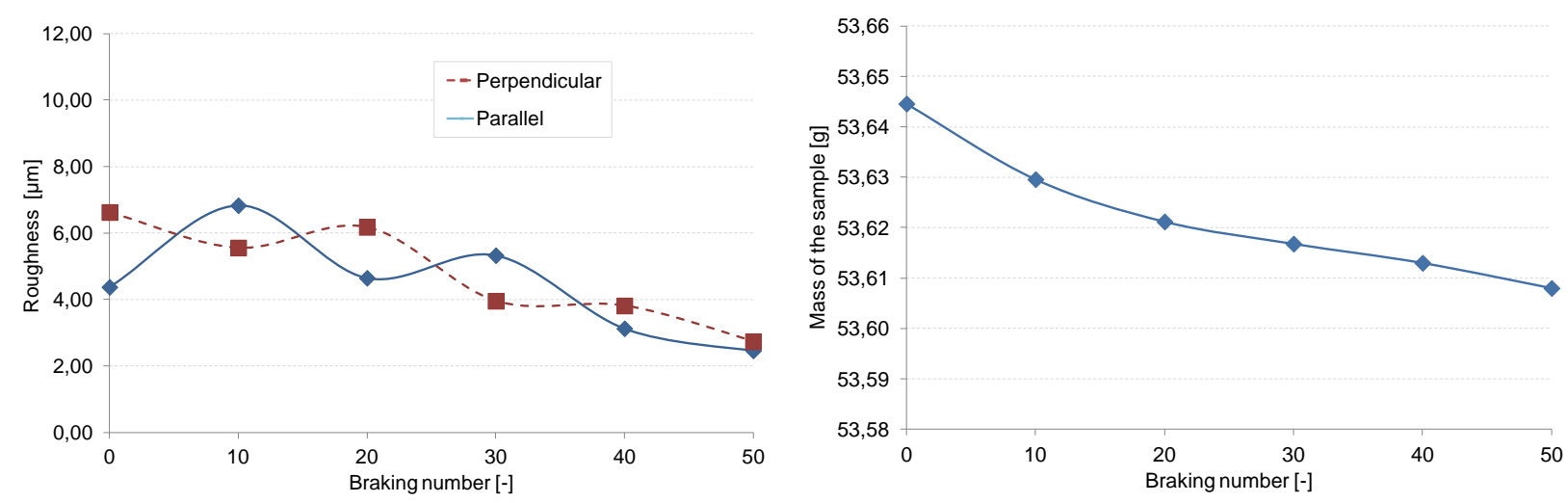

Figure 8. Roughness (left) and wear (right) evolution of the friction material.

\section{CONCLUSIONS}

The image segmentation technique was successfully applied for investigating morphological aspects related to friction material surface.

Based on the correlation results found for the NAO material selected in this study, it was concluded that:

(i) the friction behaviour is strongly associated with the amount of contact plateaus.

(ii) the size of the plateaus (mean area of plateaus) was the main factor responsible for increasing the real contact area.

The higher wear rate observed in the first braking operations can be mainly attributed to the higher surface roughness measured in this condition. As the braking operations progress, the plateaus becomes flatter and wear rate is reduced.

\section{REFERENCES}

[1] A. R. M. Lazim, M. K. A. Hamid, A. R. A. Bakar, Effects of Pad Surface Topography on Disc Brake Squeal, Applied Mechanics and Materials 165 (2012) 58-62, 2012.

[2] M. Eriksson, Friction and Contact Phenomena of Disc Brakes Related to Squeal, Doctoral thesis, Acta Universitatis Upsaliensis, Uppsala, Sweden, 2000.

[3] W. Osterle, M. Griepentrog, Th. Gross, I. Urban. Chemical and Microstructural Changes Induced by Friction and Wear of Brakes, Wear 251, 1469-1476, 2001.

[4] G.P. Ostermeyer, L. Wilkening, Experimental Investigations of the Topography Dynamics in Brake Pads, SAE International Journal Passenger Cars - Cars - Mechanical System 6 (Issue 3), 2013.

[5] P. D. Neis, Development of a Multifunctional Tribometer with Independent Temperature Control of the Brake Disk, Doctoral thesis, Joint PhD between Universidade Federal do Rio Grande do Sul (Brazil) and Ghent University (Belgium), 2012.

[6] N. Otsu, A Threshold Selection Method from Gray-Level Histograms, IEEE Transactions on Systems 9 (number 1), 1979.

[7] S. W. Yoon, M.W. Shin, W.G. Lee, H. Jang, Effect of Surface Contact Conditions on the Stick-slip Behavior of Brake Friction Material, Wear 294-295, 305-312, 2012.

[8] P.J. Blau, Compositions, Functions, and Testing of Friction Brake Materials and Their Additives, Oak Ridge National Laboratory, Technical report ORNL/TM-2001/64, Oak Ridge, US, 2001. 